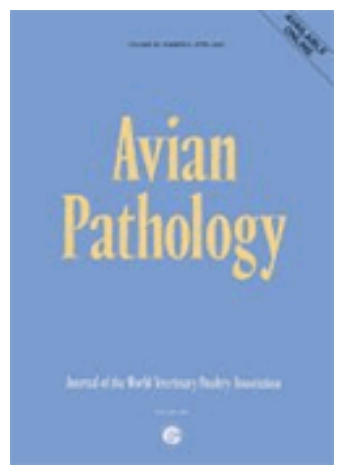

\title{
Detection and characterisation of a new astrovirus in chicken and turkeys with enteric and locomotion disorders
}

\begin{tabular}{|r|l|}
\hline Journal: & Avian Pathology \\
\hline Manuscript ID: & CAVP-2011-0071 \\
\hline Manuscript Type: & Original Research Paper \\
\hline Author: & 20-Apr-2011 \\
\hline & $\begin{array}{l}\text { Complete List of Authors: } \\
\text { Dermited, Gerdy; Intervet SPAH } \\
\text { Verstegen, Ineke; Intervet SPAH } \\
\text { Edens, Frans; GD }\end{array}$ \\
\hline Keywords: & $\begin{array}{l}\text { novel avian astrovirus, chicken turkey, enteritis runting and } \\
\text { stunting, tenosynovitis }\end{array}$ \\
\hline
\end{tabular}

\section{SCHOLARONE ${ }^{\mathrm{m}}$ Manuscripts}


Detection and characterisation of a new astrovirus in chicken and turkeys with enteric and locomotion disorders

\author{
Edens $^{1}$ and C. C. Schrier ${ }^{2 *}$, \\ ${ }^{1}$ GD Animal Health Service, P.O. Box 9, 7400 AA Deventer, \\ ${ }^{2}$ Intervet Schering-Plough Animal Health, P.O. Box 31, 5830 AA Boxmeer,
}

J.J. de Wit ${ }^{1 *}$, G.B. ten Dam², J.M.A.M. van de Laar², Y. Biermann' ${ }^{2}$ I. Verstegen ${ }^{2}$, F.

Running titile: Avian astrovirus-3 in chickens and turkeys

Received: 20 April 2011

* To whom correspondence should be addressed. Tel: +31 570 660178. E-mail:
j.d.wit@gddeventer.com; carla.schrier@sp.intervet.com 


\begin{abstract}
In this paper, we report the unexpected discovery of a new virus in samples from chicken and turkey flocks with clinical disorders such as tenosynovitis, enteric problems, or runting and/or stunting-like conditions. Since 1987, several virus isolation attempts on samples from these flocks resulted in the same macroscopic characteristic lesions in embryonated SPF eggs, being mortality with bright red discoloration of legs and wing-tips, a swollen dark-red liver and oedema. Initial work suggested presence of an agent with characteristics of a non-enveloped RNA virus. Further work, which is described in this paper, showed that the isolated strains formed a new group of avian nephritis viruses (ANV), which is genetically and antigenically distinct from known avian astroviruses (AAstVs). Inoculation of a representative strain (isolate 19) of this new group of ANV, provisionally named avian nephritis virus-3 (ANV-3), in SPF layer chicks resulted in diarrhoea, runting and stunting and even mortality.
\end{abstract}




\section{Introduction}

Astroviruses are small, round, non-enveloped, positive sense-RNA viruses measuring 28-30 $\mathrm{nm}$ in diameter. The name astrovirus comes from 'astron' (Greek for star) describing the star like morphology visualised by electron microscopy (Madeley \& Cosgrove, 1975). Astroviruses cause, in most species, problems such as gastroenteritis and growth depression. To date two different astrovirus species have been identified in chickens. Avian nephritis virus (ANV-1) was originally isolated from a 1-week-old healthy broiler chick by Yamaguchi et al. (1979) and has now been classified as an astrovirus after complete sequencing of the viral genome (Imada et al., 2000). This virus causes mild growth depression, interstitial nephritis and sometimes even mortality, especially after infection of 1-day-old SPF chickens. Most sensitive for ANV-1 are 1-day-old SPF chicks but the virus is found in all ages. Antibodies against ANV have been detected in different parts of the world, suggesting a broad distribution (Koci \& Schultz-Cherry, 2002). A second serotype of ANV (ANV-2) has been reported by several Japanese groups (Takase et al., 1989; Shirai et al.,1992).

Baxendale \& Mebatsion (2004) reported the isolation and characterisation of another astrovirus named chicken astrovirus (CAstV) which was isolated from broilers exhibiting runting and stunting-like problems. The virus and antibodies against it have been detected in broilers and broiler breeders in Europe and the USA (Baxendale \& Mebatsion, 2004; PantinJackwood et al., 2006; 2008; Day et al., 2007; Smyth et al., 2009; Todd et al., 2009b). An experimental infection of 1-day-old chicks with $\mathrm{CAstV}$ resulted in mild diarrhoea and distension of the small intestine. Recently, Todd et al. (2009a) described the identification of an antigenically and genetically different $\mathrm{CAstV}$ which had been reported earlier as enterovirus like virus (ELV) isolates FP3 and 11672 (Spackman et al., 1984). These viruses were originally isolated in the UK from flocks exhibiting early broiler mortality or hatchability problems. 
Here, we report the isolation and characterisation of a new avian astrovirus isolate that is antigenically and genetically different from the known avian or chicken astroviruses. The virus was mainly found in chicken and turkey flocks with enteric and locomotion problems. Upon infection, specific lesions were found in embryonated eggs and inoculation of 3-weekold SPF layer chickens resulted in severe interstitial nephritis, runting and stunting likeproblems and even mortality. This avian astrovirus (isolate 19), represents a novel group of ANVs, which is tentatively named avian nephritis virus-3 (ANV-3). 


\section{Materials and Methods}

Virus isolation from field samples. Collected tissues were pooled $(0.5$ to $1.0 \mathrm{~g})$, mixed and homogenised in buffer containing antibiotics. The tissues included in each sampling are indicated in Table 1 . Next, the suspension was centrifuged at $2000 \mathrm{~g}$ and subsequently filtered $(0.45 \mu \mathrm{m})$. The resulting fluid was inoculated either into the allantoic cavity of 8 or 9 dayembryonated SPF eggs, into the yolksac of 5 day-embryonated SPF eggs, or onto the chorioallantoic membrane of 9 day-embryonated SPF eggs. The inoculated eggs were incubated at $37^{\circ} \mathrm{C}$ with 40 to $60 \%$ humidity and were candled daily. Embryonic death occurring within one day of inoculation was considered to be non-specific. Up to 5 passages were made. In Table 1 a description is found of 10 isolates selected from a large number collected over the years. The representative isolate of this group is referred to as isolate 19 .

Titration on embryonated chicken eggs. Serial 10-fold dilutions of the suspension of isolate 19 were inoculated into the yolk sac of six-day-old embryonated SPF chicken eggs. Subsequently, the eggs were incubated for 7 days at $37^{\circ} \mathrm{C}$ and $40-60 \%$ humidity. Mortality occurring within $24 \mathrm{~h}$ was considered non-specific. Embryos dying from 2 to 7 days post inoculation (d.p.i) were inspected for the presence of specific lesions. The infectivity titre calculation was based on the method of Spearman \& Kärber (Finney, 1952) and was expressed as median embryo infective dose $\left(\mathrm{EID}_{50}\right)$ per $\mathrm{ml}$.

RNA extraction and RT-PCR analysis. Viral RNA was extracted using QIAamp ${ }^{\circledR}$ Viral RNA Mini Kit (Qiagen) and reverse transcription (RT) was performed using Ready-To-Go ${ }^{\mathrm{TM}}$ You-Prime First-Strand Beads (GE Healthcare), both according to the manufacturer's instructions. For PCR $2 \mu 1$ of cDNA was used in a 96-well thermal cycler (Mastercycler 
Gradient, Eppendorf). The reaction mixtures contained $1 \mu \mathrm{m}$ forward and reverse primers

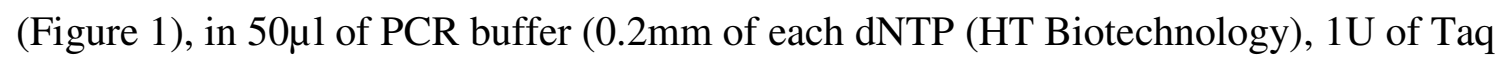
DNA polymerase (HT Biotechnology), 10mM Tris- $\mathrm{HCl}$ (pH 9.0), 1.5mM $\mathrm{MgCl}_{2}, 50 \mathrm{mM} \mathrm{KCl}$, $0.1 \%$ Triton X100 and $0.01 \%(\mathrm{w} / \mathrm{v})$ gelatin). The PCR program included: $30 \mathrm{sec}$ at $95^{\circ} \mathrm{C}$ followed by 45 cycles of $30 \mathrm{sec}$ at $95^{\circ} \mathrm{C}, 30 \mathrm{sec}$ annealing temperature (Figure 1) and $40 \mathrm{sec}$ at $72^{\circ} \mathrm{C}$, followed by $10 \mathrm{~min}$ at $72^{\circ} \mathrm{C}$. For the nested PCR, a similar PCR was performed using $2 \mu \mathrm{l}$ of PCR product and 30 PCR cycles. The PCR products were visualized by agarose gel electrophoresis and purified using the QIAquick ${ }^{\circledR}$ Gel Extraction kit (QiaGen ${ }^{\mathrm{TM}}$ ) according to the manufacturer's instructions.

DNA sequencing. DNA sequencing was performed by standard PCR cycle-sequencing, using a Big Dye ${ }^{\circledR}$ Terminator Ready Reaction Mix (ABI Prism ${ }^{\mathrm{TM}}$ ) and an ABI Prism ${ }^{\mathrm{TM}} 310$ automated sequencing apparatus, all according to the manufacturer's instructions. Typically 20 to 70ng of DNA (purified PCR product) was used in a reaction with $8 \mu 1$ Big Dye ${ }^{\circledR}$ Terminator Ready Reaction Mixture and $0.5 \mu \mathrm{m}$ primer in $20 \mu 1$. The primers used for sequencing were the forward F-II (5'-AAA GGK AAG ACD AAG ARR RAC MG-3)' and the reverse R-II-3 (5'- TCG CCT TCT GGA AGG TCT TCA-3') primers, which amplified a fragment of 469 bp that corresponded to nucleotides 2171-2640 of the ANV1 G4260 genome. The cycle sequencing PCR program contained 25 cycles of: $10 \mathrm{sec}$ at $96^{\circ} \mathrm{C}, 5 \mathrm{sec}$ at $50^{\circ} \mathrm{C}$ and 4 min at $60^{\circ} \mathrm{C}$. Samples were purified using DyeEx ${ }^{\circledR}$ Spin columns (QiaGen ${ }^{\mathrm{TM}}$ ), according to the manufacturer's instructions and resuspended in $40 \mu 1$. Sequence determination was performed using ABI Prism ${ }^{\circledR} 310$ Genetic Analyzer with Data Collection version 1.0.4 en Sequence Analysis version 3 software. 
Inoculation of chickens. An animal experiment was conducted to (1) attempt to reproduce the disease signs found in the field and re-isolate the virus (compliance with Koch's postulates), (2) to observe whether the virus would spread to sentinel birds and (3) to produce polyclonal antiserum against the inoculated avian astrovirus. For these purposes, 20 individually labeled 3-week-old SPF layer type chickens (both sexes) were transferred to a negative pressure isolator. Standard food and drinking water were available ad libitum. Fifteen chickens were inoculated with embryo homogenate of isolate 19 by three routes: 0.2 $\mathrm{ml}$ each by the ocular and intramuscular routes $\left(10^{5} \mathrm{EID}_{50} / 0.2 \mathrm{ml}\right)$, as well as $0.5 \mathrm{ml}\left(10^{5.4}\right.$ $\mathrm{EID}_{50}$ ) by the oral route. The remaining 5 chickens were not inoculated and served as sentinels to observe spreading of the virus. Animals were inspected daily for clinical signs of disease and/or mortality. At 7 d.p.i. 5 chickens were bled and submitted for post-mortem (PM) and histological examination (Table 2). At necropsy, samples of duodenum, liver, thymus, kidney, tendon, bursa and pancreas were taken for histological examination. Kidney samples were also used for detection by RT-PCR. PM and histological examination was also performed on chicks that showed severe illness or died during the experiment. The remaining (inoculated) birds were boosted at 37d.p.i. and bled at 58 d.p.i. The sera were pooled and used as antiserum in the immunofluorescence assay.

Immunofluorescence assay. Microtitration plates containing chicken embryo kidney (CEK), chicken embryo liver (CEL) or LMH cells [a chicken hepatoma cell line] (Kawaguchi, et al., 1987) were inoculated with isolate 19 , ANV1 or CAstV. After incubation at $37^{\circ} \mathrm{C}$ in $5 \% \mathrm{CO}_{2}$ atmosphere for two days, the plates were fixed and incubated with dilutions of the chicken antisera raised against the different astrovirus strains, chicken serum samples from the field, or PBS as negative control. Following incubation for $1 \mathrm{~h}$ at $37^{\circ} \mathrm{C}$ in a moist atmosphere, the plates were washed with PBS. One hour after incubation with goat anti-chicken IgG-FITC 
conjugate (Nordic ${ }^{\mathrm{TM}}$ ) the plates were washed with PBS and mounting buffer was added (PBS/glycerol). A positive signal was the detection of fluorescence, correlating with specific antibodies against the virus-infected cells.

Seroprevalence field study. A small-scale seroprevalence study was performed by testing sera from 10 broiler flocks and 12 layer flocks that were chosen randomly from the routine submissions to the GD Animal Health Service Laboratory. No clinical data were available for these flocks. From each flock, 7 to 30 blood samples were collected and tested for the presence for specific antibodies by immunofluorescence against ANV-1, CAstV and isolate 19 in virus infected LMH cells or chicken embryo liver (CEL) cells.

\section{Results}

Virus isolation from field samples and initial typing. Since 1987, an unknown agent has been isolated from a variety of clinical disorders from chicken and turkey flocks in The Netherlands, Germany and the United Arab Emirates (Table 1). Most of these flocks were broiler or turkey flocks with enteric problems and/or arthritis and tenosynovitis resulting in diarrhoea, reduction in feed conversion and growth, and/or swelling and discomfort of the joints (especially the hock) and tendons of the legs resulting in considerable economic damage. The unknown agent had also been isolated from sick broiler breeder and layer flocks.

In most cases involvement of a reovirus was suspected as this virus is well known for causing malabsorption, arthritis and tenosynovitis. However, the isolates were neither reovirus nor any other known pathogen including adenovirus, infectious bursal disease virus, infectious bronchitis virus, paramyxovirus, orthomyxovirus, infectious laryngotracheitis virus, 
and chicken anaemia virus (data not shown). Also, bacteria including mycoplasma could be excluded. The isolates caused characteristic macroscopic lesions in SPF embryos, namely bright red discoloration of legs and wing-tips, a swollen dark-red liver and oedema. As the embryo abnormalities did not decrease with further passages, a toxic agent could also be excluded. Treatment of these isolates with high concentrations of acid and chloroform did not influence the infectivity. It was concluded that the infectious agent was most likely a nonenveloped RNA virus.

Identification of the novel avian astrovirus. PCR and sequence analysis experiments were performed to identify the infectious agent present in the field samples. Positive PCR results were obtained using the degenerate general astrovirus specific RT primer 17 (Figure 1) in combination with primer sets $20 / 21$ and $23 / 24$ to detect respectively all avian astroviruses or more specifically the ANVs. These results indicated that isolate 19, and all other strains as listed in Table 1, belonged to the family of Astroviridae. However, the PCR was negative using primer set 27/28 specific for chicken astroviruses, indicating that isolate 19 and the other field strains were genetically different from the CAstV strains as described by Baxendale \& Mebatsion (2004).

A specific PCR was developed after analysis of the sequence data of isolate 19 and the other field strains (see below). This PCR, using primers 29 and 30, gave positive results with samples of all field isolates mentioned in Table 1, whereas RT-samples prepared from ANV1 and CAstV were consistently found to be negative. The sizes of these PCR products were approximately $260 \mathrm{bp}$.

The 10 strains were sequenced and aligned using as reference the sequence of isolate 19. Sequences of the corresponding parts of ORF 1a from other astroviruses available in GenBank were also aligned, for instance ANV1, turkey astrovirus 1 (TAstV1), turkey 
astrovirus 2 (TAstV2), sheep astrovirus, mink astrovirus and human astrovirus (GenBank accession numbers are listed in Table 1). Only ANV1 (nt 2207 - 2607) showed a significant nucleotide sequence identity of $80 \%$ (Table 1). Of the other astroviruses, only TAstV1 had a detectable, but hardly significant, nucleotide sequence identity of $56 \%$. The sequence homology of the other 9 field strains varied from 88 to $98 \%$ compared to the sequence of isolate 19. When all field strains were compared with ANV1 (Table 1) a sequence homology of $80-82 \%$ was observed between each of the new isolates and ANV1. A multiple alignment, using isolate 19 as a reference, of nucleotide sequences and deduced amino acid sequences of the ORF 1a region of the 10 isolates and ANV1 is listed in Figures 2 and 3 respectively.

Remarkably, an insert of 12 nucleotides in the ORF 1a, present in all 10 field strains, distinguished these novel AAstVs from ANV1 and other types of known astroviruses. For all 10 isolates, the 12 nucleotide insert in ORF 1a was located between nucleotides corresponding to the nucleotides 2485 and 2486 of the reference ANV1 strain (Imada et al., 2000). The nucleotide sequence of the 12 nucleotide insert was not identical for all the isolates, however size and location were. The consensus of the nucleotide sequence of the 12 nucleotide insert was: 5' - TCYGGDMARYYT -3'. A dendrographic tree of the results of these nucleotide sequence alignments is presented in Figure 4.

Infection of chickens by the novel avian astrovirus. Inoculation of isolate 19 into 3 -weekold SPF layer chickens resulted in clear clinical signs, macroscopical and histopathological lesions, detection of the astrovirus in the kidneys of the chickens and development of an antibody response. The clinical signs observed were diarrhoea (1 inoculated, 1 sentinel bird) and severe depression ( 2 inoculated, 2 sentinel birds). In total 2 chickens died ( 1 inoculated bird at 21 d.p.i., 1 sentinel bird at day 28), and another 2 severely ill birds (1 inoculated, 1 sentinel) were killed humanely for animal welfare reasons at day 23. Histopathology was 
performed on 5 birds at 7 d.p.i. and all the birds that died during the experiment (Table 2). The kidneys of these birds showed severe interstitial nephritis and tubular degeneration (7/8) as the most prominent signs The villi in the duodenum were blunted and fused (9/9) and the thymus and bursa showed lymphocytolysis and apoptosis (8/9). Acinar atrophy (7/9) was detected in the pancreas; no abnormalities were observed in the tendon. Horizontal infection to sentinel birds that showed disease signs demonstrated the virulence and infectivity of astrovirus isolate 19. The kidney samples of all 5 inoculated birds (Table 2) were RT-PCR positive at 7 d.p.i., demonstrating the presence of the virus in the kidneys of the inoculated birds.

Immunological relationship between ANV1, CAstV and isolate 19. CEK or CEL cells were infected with the novel avian astrovirus isolate 19, ANV1 and CAstV and the immunological cross-reactivity with the chicken antisera raised against isolate 19, ANV1 and $\mathrm{CAstV}$ was investigated. The results of these immunofluorescence assays are listed in Table 3. Each of the 3 antisera showed high titres of $>12 \log _{2}$ to the specific astrovirus species they had been raised against and no or only very minor cross-reactivity (all titres $<5 \log _{2}$ ) against the other astroviruses.

Seroprevalence field survey. The results of the small scale field survey for the presence of isolate 19, ANV1 and CAstV specific antibodies in serum samples from broiler and layer flocks from The Netherlands with no or unknown clinical signs are listed in Table 4. Antibodies specific for ANV1 were found in 1 broiler flock and 4 layer flocks, whereas antibodies specific for isolate 19 were present in a high number of broiler (8/10) as well as layer (7/12) flocks. Birds positive for ANV1 were not necessarily positive for isolate 19. In all 
flocks tested, antibodies specific for CastV were present and in some cases more than $80 \%$ of the birds tested were positive.

\section{Discussion}

In this paper, we report the discovery of a new astrovirus present in chicken and turkey flocks with clinical disorders such as arthritis, tenosynovitis, enteric problems, or runting and/or stunting-like conditions. Strains of this formerly unknown virus caused the same, macroscopic, characteristic lesions upon infection of embryonated SPF eggs, being mortality with bright red discoloration of legs and wing-tips, a swollen dark-red liver and oedema. The presented work showed that these strains formed a new type of AAstVs, which is genetically and antigenically distinct from known AAstVs.

The genetic information of 10 of these strains showed that the closest (yet distinguishable) relatives were ANV-1 (80-82\% nucleotide sequence similarity in nucleotides 2207 - 2607 of ORF1a) and TAstV1 (about 56\% nucleotide sequence similarity in nucleotides $2450-2856$ of ORF1a). The presence of an insert of 12 nucleotides in the ORF 1a, present in all 10 isolates, distinguished the novel AAstVs from ANV-1 reference strain G4260 and any other type of known astrovirus. Further work is needed to reveal the association of the 12 nucleotide insert in ORF 1a and the immunological and pathobiological differences observed between these groups of astroviruses. The fact that the insert consists of 12 nucleotides, thus 4 triplets which does not disturb the translational frame, suggests that the encoded 4 additional amino acids may have a role in the non-structural proteins that are expressed by ORF 1a. Independently from its biological meaning, the insert can be used as a diagnostic, positive 
genetic marker for this novel group of viruses. However, it would be very useful to acquire more knowledge about the ORF1a sequence of more ANV-1 strains.

The antigenicity of isolate 19 of the novel group of ANVs was markedly different from ANV-1 and CAstV as was shown by the immunofluorescence analysis. The antiserum directed against the representative isolate 19 of the novel group was not recognized by ANV1 or CAstV and vice versa. At this moment, the antigenic relationship between isolate 19 and each of the other 9 strains detected here has yet to be investigated. Therefore it cannot yet be excluded that the new genetic group of ANVs could consist of multiple antigenic types. Further work is needed on this aspect.

Isolate 19 of the new group of ANVs, which has mainly been detected in chickens and turkeys suffering from runting and stunting like and/or locomotory disease, induced similar signs of disease upon inoculation of healthy 3-week-old SPF layer chickens, namely diarrhoea and runting and stunting like symptoms, and could be detected by RT-PCR from the diseased chickens, thereby fulfilling Koch's postulates. The clinical tenosynovitis, which was seen regularly in the broilers and poults from the field was not seen in the experimentally infected 3-week-old SPF layers. The isolation of these new avian astrovirus strains from chickens and turkeys with acute tenosynovitis and arthritis without the isolation of any other detectable pathogen suggests that they also might play a role in leg disorders. Possibly the outcome of the experimental infection was influenced by the type of chickens used in this experiment, as the acute tenosynovitis and arthritis leg disorders were all from broilers and turkeys. Another possible factor could be the difference in the conditions of SPF chickens in an isolator and the multiple infective pressure obtained under field conditions. Therefore, more experiments are needed to clarify the role of these AAstVs in tenosynovitis and arthritis.

Isolate 19 also spread from the inoculated to the sentinel birds, showing its virulence and infectivity. The pathogenicity of isolate 19, as a representative of the novel astrovirus, 
was further demonstrated by the mortality and histopathological findings of the infected 3week-old SPF birds, which were much more severe than those described for infections with CAstV strains. Experimental infections of 1-day-old and 5-day-old SPF layer chickens with CAstV caused mild diarrhoea and except for some areas of limited damage at the base of the villi of the small intestine in some birds, no histological visible damage in liver, kidney, spleen, thymus or bursa was observed (Baxendale \& Mebatsion, 2004). McNeilly et al. (1994) used enterovirus-like virus ELV-4 strain 612, later (Todd et al., 2009a) typed as a CAstV closely related to the isolate of Baxendale \& Mebatsion (2004), to infect commercial 1-day-old broilers with maternal antibodies against the virus and found only a growth depression of $11.3,20.4$ and $9.6 \%$ respectively in the three trials that were performed. Experimental infections of 1-day-old SPF layer chickens with CAstV strain 612 caused only very mild histological changes in the intestine and liver, and mild to moderate lesions in the kidney (Smyth et al., 2007). Experimental infections of 1-day-old SPF layer chickens with CAstV strain FP3 that is genetically and antigenically distinguishable from strain 612 (Todd et al., 2009a), originally known as ELV-3, (McNulty et al., 1990) caused only mild to moderate histological changes in the intestine, pancreas and kidney (Smyth et al., 2007). An experimental infection with the FP3 strain caused no clinical signs in 1-day-old broilers (McNulty et al., 1990) with an unknown level of maternal antibodies against FP3.

The pathogenicity of isolate 19 seems to be broader than is reported for ANV-1. Many studies have shown that the pathogenicity of ANV-1 is limited to the kidney of chickens, in contrast to the damage observed in kidney, pancreas, duodenum, thymus, bursa and liver that was seen after infection with isolate 19 (Imada, 2008). This pathogenicity of isolate 19 was observed after infection of 3-week-old SPF layers, whereas several strains of ANV-1 caused considerable mortality in 1-day-old SPF chicks but not in older birds (Imada et al., 1981; Frazier et al., 1990; Narita et al., 1990; Shirai et al., 1991a; Shirai et al., 1991b; Reece et al., 
1992; Shirai et al., 1992). These differences can be explained by the different type of ANV strains, by strain of bird, route of inoculation or level of maternally derived antibodies; other experimental conditions should also be taken into account (Mandoki et al., 2006; Reynolds \& Schultz-Cherry, 2008). It would also be very interesting to compare the pathogenicity of more strains of the ANV-3 group.

Further work is needed to determine the prevalence and relevance of this new group of AAstVs for the international poultry industry. Almost all isolates so far tested were obtained from Dutch and German chickens and turkeys, with the interesting exception of one isolate from the United Arab Emirates. The virus has been detected almost every year since 1987 from clinically affected Dutch and/or German poultry which could indicate that the virus might well be of significance. A limited field survey in The Netherlands indicated that antibodies specific for isolate 19 are found regularly in broiler as well as layer flocks, suggesting that a field infection has occurred with a virus related to isolate 19. Regarding the resistant nature of astroviruses, it would not be surprising if the virus were to be present in more areas of the international poultry industry, as has been shown for other AAstVs.

\section{References}

Baxendale, W. \& Mebatsion, T. (2004). The isolation and characterisation of astroviruses from chickens. Avian Pathology, 33, 364-370.

Day, J. M., Spackman, E. \& Pantin-Jackwood, M. (2007). A multiplex RT-PCR test for the differential identification of turkey astrovirus type 1, turkey astrovirus type 2 , chicken astrovirus, avian nephritis virus, and avian rotavirus. Avian Diseases, 51, 681-684.

Finney, D. J. (1952). In: Statistical Method in Biological Assay (pp 524-530). Charles Griffin (Ed.), London. 
Frazier, J. A., Howes, K., Reece, R. L., Kidd, A. W. \& Cavanagh, D. (1990). Isolation of noncytopathic viruses implicated in the aetiology of nephritis and baby chick nephropathy and serologically related to avian nephritis virus. Avian Pathology, 19, 139-160.

Imada, T. (2008). Avian Nephritis. In: Saif, Y.M., Fadly, A.M., Glisson, J.R., McDougald, L.R., Nolan, L.K. and Swayne, D.E. (Eds.), Diseases of Poultry (12th ed., pp. 409413): Blackwell Publishers.

Imada, T., Taniguchi, T., Yamaguchi, S., Minetoma, T., Maeda, M. \& Kawamura, H. (1981). Susceptibility of chickens to avian nephritis virus at various inoculation routes and ages. Avian Diseases, 25, 294-302.

Imada, T., Yamaguchi, S., Mase, M., Tsukamoto, K., Kubo, M. \& Morooka, A. (2000). Avian nephritis virus (ANV) as a new member of the family Astroviridae and construction of infectious ANV cDNA. Journal of Virology, 74, 8487-8493.

Kawaguchi, T., Nomura, K., Hirayama, Y. \& Kitagawa, T. (1987) Establishment and characterization of a chicken hepatocellular carcinoma cell line, LMH. Cancer Research, 47, 4460-4464.

Koci, M. D. \& Schultz-Cherry, S. (2002). stVs. Avian Pathology, 31, 213-227.

Madeley, C. R. \& Cosgrove, B. P. (1975). Letter: $28 \mathrm{~nm}$ particles in faeces in infantile gastroenteritis. Lancet, 2, 451-452.

Mandoki, M., Bakonyi, T., Ivanics, E., Nemes, C., Dobos-Kovacs, M. \& Rusvai, M. (2006). Phylogenetic diversity of avian nephritis virus in Hungarian chicken flocks. Avian Pathology, 35, 224-229.

McNulty, M. S., Connor, T. J., McNeilly, F. \& McFerran, J. B. (1990). Biological characterisation of avian enteroviruses and enterovirus-like viruses. Avian Pathology, $19,75-87$. 
Narita, M., Ohta, K., Kawamura, H., Shirai, J., Nakamura, K. \& Abe, F. (1990). Pathogenesis of renal dysfunction in chicks experimentally induced by avian nephritis virus. Avian Pathology, 19, 571-582.

Pantin-Jackwood, M. J., Spackman, E. \& Woolcock, P. R. (2006). Molecular characterization and typing of chicken and turkey astroviruses circulating in the United States: implications for diagnostics. Avian Diseases, 50, 397-404.

Pantin-Jackwood, M. J., Day, J. M., Jackwood, M. W. \& Spackman, E. (2008). Enteric viruses detected by molecular methods in commercial chicken and turkey flocks in the United States between 2005 and 2006. Avian Diseases, 52, 235-244.

Reece, R. L., Howes, K. \& Frazier, J. A. (1992) Experimental factors affecting mortality following inoculation of chickens with avian nephritis virus (G-4260). Avian Diseases, $36,619-624$.

Reynolds, D. L. \& Schultz-Cherry, S. L. (2008). Astrovirus Infections. In: Saif, Y.M., Fadly, A.M., Glisson, J.R., McDougald, L.R., Nolan, L.K. and Swayne, D.E. (Eds.), Diseases of Poultry (12th ed., pp. 351-355): Blackwell Publishers.

Shirai, J., Nakamura, K., Nozaki, H. \& Kawamura, H. (1991a). Differences in the induction of urate deposition of specific-pathogen-free chicks inoculated with avian nephritis virus passaged by five different methods. Avian Diseases, 35, 269-275.

Shirai, J., Nakamura, K., Shinohara, K. \& Kawamura, H. (1991b). Pathogenicity and antigenicity of avian nephritis isolates. Avian Diseases, 35, 49-54.

Shirai, J., Tanimura, N., Uramoto, K., Narita, M., Nakamura, K. \& Kawamura, H. (1992). Pathologically and serologically different avian nephritis virus isolates implicated in etiology of baby chick nephropathy. Avian Diseases, 36, 369-377. 
Smyth, J. A., Connor, T. J., McNeilly, F., Moffet, D. A., Calvert, V. M. \& McNulty, M. S. (2007). Studies on the pathogenicity of enterovirus-like viruses in chickens. Avian Pathology, 36, 119-126.

Smyth, V. J., Jewhurst, H. L., Adair, B. M. \& Todd, D. (2009). Detection of chicken astrovirus by reverse transcriptase-polymerase chain reaction. Avian Pathology, 38, 293-299.

Spackman, D., Gough, R. E., Collins, M. S. \& Lanning, D. (1984). Isolation of an enterovirus-like agent from the meconium of dead-in-shell chicken embryos. Veterinary Record, 114, 216-218.

Takase, K., Shinohara, K., Tsuneyoshi, M., Yamamoto, M. \& Yamada, S. (1989). Isolation and characterisation of cytopathic avian enteroviruses from broiler chicks. Avian Pathology, 18, 631-642.

Todd, D., Smyth, V. J., Ball, N. W., Donnelly, B. M., Wylie, M., Knowles, N. J. \& Adair, B. M. (2009a). Identification of chicken enterovirus-like viruses, duck hepatitis virus type 2and duck hepatitis virus type 3 as astroviruses. Avian Pathology, 38, 21-30.

Todd, D., Wilkinson, D. S., Jewhurst, H. L., Wylie, M., Gordon, A. W. \& Adair, B. M. (2009b). A seroprevalence investigation of chicken astrovirus infections. Avian Pathology, 38, 301-309.

Yamaguchi, S., Imada, T. \& Kawamura, H. (1979). Characterization of a picornavirus isolated from broiler chicks. Avian Diseases, 23, 571-581. 
Table 1. Characteristics of 10 new avian nephritis virus isolates compared to ANV-1 and other astrovirus species.

\begin{tabular}{|c|c|c|c|c|c|c|c|c|c|c|}
\hline \multirow{2}{*}{$\begin{array}{l}\text { Isolate } \\
\text { reference }\end{array}$} & \multicolumn{4}{|c|}{ Data on flocks from which isolates were obtained } & \multirow{2}{*}{$\begin{array}{l}\text { Year of } \\
\text { isolation }\end{array}$} & \multicolumn{3}{|c|}{$\%$ sequence identity (ORF1a) } & \multirow{2}{*}{$\begin{array}{c}\text { Presence of } 4 \\
\text { amino acid } \\
\text { insert }\end{array}$} & \multirow[b]{2}{*}{ Genbank accession number } \\
\hline & Species & Type & Age & Tissue & & $\begin{array}{l}\text { nt }^{\mathrm{a}} \text { (with } \\
\text { ANV1) }\end{array}$ & $\begin{array}{l}\mathrm{nt}^{\mathrm{b}} \text { (with } \\
\text { isolate19) }\end{array}$ & $\begin{array}{l}\mathrm{AA}^{\mathrm{c}} \text { (with } \\
\text { isolate19) }\end{array}$ & & \\
\hline 19 & Chicken & Layer & 36 weeks & Trachea & 2004 & 80 & 100 & 100 & Yes & FJ940720 \\
\hline 637 & Chicken & broiler & 12 days & Tendon and hock-cartilage & 2005 & 82 & 90 & 97 & Yes & FJ959032 \\
\hline 686 & Chicken & broiler & 10 days & Various organs & 2005 & 80 & 88 & 97 & Yes & FJ959035 \\
\hline 714 & Chicken & broiler & 30 days & Caecal tonsils and pancreas & 2005 & 82 & 89 & 97 & Yes & FJ959036 \\
\hline 715 & Chicken & broiler & 30 days & Caecal tonsils and pancreas & 2005 & 80 & 98 & 99 & Yes & FJ959037 \\
\hline 1736 & Chicken & broiler & 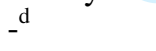 & Tendon and tibia & 1987 & 81 & 89 & 96 & Yes & FJ959038 \\
\hline 2383 & Turkey & poults & - & Joint and synovial fluid & 1988 & 81 & 88 & 94 & Yes & FJ959033 \\
\hline 2388 & Turkey & poults & - & Tendon and tibia & 1988 & 80 & 88 & 93 & Yes & FJ959034 \\
\hline 7279 & Chicken & -- & - & Pancreas & 2000 & 82 & 89 & 96 & Yes & FJ959039 \\
\hline 161317 & Chicken & Layer & 36 weeks & Trachea & 2004 & 81 & 89 & 97 & Yes & FJ959040 \\
\hline $\mathrm{ANV}-1^{\mathrm{e}}$ & & & & & & 100 & 80 & 88 & No & AB033998 (nt: 2207-2607) \\
\hline TAstV1 & & & & & & $N D^{f}$ & 56 & $\mathrm{Nd}$ & No & Y15936 (nt: 2450-2856) \\
\hline CAstV & & & & & & & & & & EU669000 \\
\hline TAstV2 & & & & & & & & & & AF206663 \\
\hline SAstV & & & & & & ND & $<50$ & $<40$ & No & Y15937 \\
\hline MAstV & & & & & & & & & & AY179509 \\
\hline HAstV1 & & & & & & & & & & Z25771 \\
\hline
\end{tabular}

${ }^{a}$ Nucleotide sequence identity (ORF1a) between ANV1 and the 10 isolates.

${ }^{\mathrm{b}}$ Nucleotide sequence identity between isolate 19 (ORF1a) and the other isolates.

${ }^{c}$ Amino acid sequence identity between isolate 19 (ORF1a) and the other isolates and other known astroviruses.

d - : not known.

${ }^{\mathrm{e}} \mathrm{ANV}-1=$ avian nephritis virus $1, \mathrm{TAstV} 1=$ turkey astrovirus $1, \mathrm{TAstV} 2=$ turkey astrovirus $2, \mathrm{SAst} \mathrm{V}=$ sheep astrovirus, $\mathrm{MAst} \mathrm{V}=$ mink astrovirus and $\mathrm{HAstV}=$ human astrovirus.

${ }^{\mathrm{f}}$ Not determined. 
Table 2. Clinical signs, histopathology, and ANV-3 RT-PCR results after inoculation of isolate 19 (avian nephritis virus type 3) in 3-week-old SPF birds

\begin{tabular}{|c|c|c|c|c|c|c|}
\hline \multicolumn{5}{|c|}{ Histopathology results for 5 birds killed at 7 d.p.i. ${ }^{a}$ and birds that died during the experiment } & \multicolumn{2}{|c|}{ istology results for 5 sentinel birds } \\
\hline Organ & Histopathology & $\begin{array}{c}5 \text { killed } \\
7 \text { d.p.i } \\
\end{array}$ & $\begin{array}{l}1 \text { died } \\
21 \text { d.p.i. }\end{array}$ & $\begin{array}{l}1 \text { killed }^{b} \\
23 \text { d.p.i. }\end{array}$ & $\begin{array}{l}1 \text { killed }^{b} \\
23 \text { d.p.i. }\end{array}$ & $\begin{array}{l}1 \text { died } \\
28 \text { d.p.i. }\end{array}$ \\
\hline Kidney & $\begin{array}{c}\text { Severe interstitial } \\
\text { nephritis and tubular } \\
\text { degeneration }\end{array}$ & $5 / 5^{\mathrm{c}}$ & $1 / 1$ & $0 / 1$ & $1 / 1$ & $\mathrm{NT}^{\mathrm{d}}$ \\
\hline Pancreas & Apoptosis & $5 / 5$ & $0 / 1$ & $0 / 1$ & $0 / 1$ & $1 / 1$ \\
\hline & Acinar atrophy & $5 / 5$ & $1 / 1$ & $0 / 1$ & $0 / 1$ & $1 / 1$ \\
\hline Duodenum & Blunted and fused villi & $5 / 5$ & $1 / 1$ & $1 / 1$ & $1 / 1$ & $1 / 1$ \\
\hline Thymus & Lymphocytolysis & $4 / 5$ & $1 / 1$ & $1 / 1$ & $1 / 1$ & $1 / 1$ \\
\hline Bursa & Lymphocytolysis & $5 / 5$ & $1 / 1$ & $1 / 1$ & $1 / 1$ & $1 / 1$ \\
\hline Liver & $\begin{array}{c}\text { Multifocally } \\
\text { mononuclear aggregation }\end{array}$ & $5 / 5$ & $1 / 1$ & NT & $1 / 1$ & $0 / 1$ \\
\hline Tendon & Abnormalities & NT & NT & $0 / 1$ & $0 / 1$ & $0 / 1$ \\
\hline Kidney & ANV-3 RT-PCR ${ }^{\mathrm{e}}$ & $5 / 5$ & $0 / 1$ & $0 / 1$ & $0 / 1$ & $0 / 1$ \\
\hline
\end{tabular}

\footnotetext{
${ }^{a}$ Days post infection.

${ }^{\mathrm{b}}$ Humanely killed for animal welfare reasons.

${ }^{\mathrm{c}}$ Number affected/number examined.

${ }^{\mathrm{d}}$ Not tested.

${ }^{\mathrm{e}} \mathrm{RT}$-PCR specific for avian nephritis virus-3.
} 
Table 3. Immunological relationship between the astroviruses ANV-1, CAstV and isolate 19.

\begin{tabular}{ccccc}
\hline \multirow{2}{*}{ Antiserum } & \multicolumn{4}{c}{ Immunofluorescence assay with viruses } \\
& ANV1 & CASTV & Isolate 19 & CEK/CEL $^{\text {a }}$ \\
\hline anti-ANV-1 & $>12^{\mathrm{b}}$ & $<5$ & $<5$ & $<5$ \\
anti-CAstV & $<5$ & $>12$ & $<5$ & $<5$ \\
anti-isolate 19 & $<5$ & $<5$ & $>12$ & $<5$ \\
SPF serum & $<5$ & $<5$ & $<5$ & $<5$ \\
\hline
\end{tabular}

${ }^{\text {a }}$ None infected cells.

${ }^{\mathrm{b}}$ Reciprocal titres in the immunofluorescence assay expressed as $\log 2$. 
Table 4. Detection by immunofluorescence of antibodies to ANV-1, CAstV and ANV-3 isolate 19

in serum samples collected from broiler and layer flocks with no, or unknown, clinical signs in The Netherlands in 2009.

\begin{tabular}{llllll}
\hline \multirow{2}{*}{ Flock type } & \multirow{2}{*}{ Flock } & \multicolumn{2}{l}{ Age in days (d) or } & \multicolumn{3}{l}{ Antibodies detected to } \\
& & weeks (w) & ANV-1 & CAstV & Isolate 19 \\
\hline Broiler & 1 & $44 \mathrm{~d}$ & $1 / 30(3 \%)^{\mathrm{a}}$ & $19 / 30(63 \%)$ & $6 / 30(20 \%)$ \\
Broiler & 2 & $34 \mathrm{~d}$ & $0 / 24$ & $13 / 22(59 \%)$ & $1 / 27(4 \%)$ \\
Broiler & 3 & $41 \mathrm{~d}$ & $0 / 9$ & $1 / 7 \quad(17 \%)$ & $4 / 10(40 \%)$ \\
Broiler & 4 & $41 \mathrm{~d}$ & $0 / 23$ & $8 / 22(36 \%)$ & $0 / 25$ \\
Broiler & 5 & $41 \mathrm{~d}$ & $0 / 29$ & $14 / 30(47 \%)$ & $0 / 30$ \\
Broiler & 6 & $36 \mathrm{~d}$ & $0 / 26$ & $12 / 25(48 \%)$ & $6 / 27(22 \%)$ \\
Broiler & 7 & $35 \mathrm{~d}$ & $0 / 30$ & $24 / 30(80 \%)$ & $2 / 30(7 \%)$ \\
Broiler & 8 & $38 \mathrm{~d}$ & $0 / 25$ & $11 / 24(46 \%)$ & $4 / 25(16 \%)$ \\
Broiler & 9 & $38 \mathrm{~d}$ & $0 / 28$ & $10 / 28(36 \%)$ & $11 / 28(39 \%)$ \\
Broiler & 10 & $40 \mathrm{~d}$ & $0 / 21$ & $18 / 22(82 \%)$ & $1 / 30(3 \%)$ \\
Layer & $\mathrm{A}$ & $29 \mathrm{w}$ & $0 / 14$ & $8 / 14(57 \%)$ & $2 / 14(14 \%)$ \\
Layer & $\mathrm{B}$ & $23 \mathrm{w}$ & $0 / 14$ & $8 / 14(57 \%)$ & $0 / 14$ \\
Layer & $\mathrm{C}$ & $29 \mathrm{w}$ & $2 / 15(13 \%)$ & $12 / 15(80 \%)$ & $0 / 14$ \\
Layer & $\mathrm{D}$ & $28 \mathrm{w}$ & $1 / 12(8 \%)$ & $3 / 12(25 \%)$ & $0 / 12$ \\
Layer & $\mathrm{E}$ & $28 \mathrm{w}$ & $0 / 10$ & $7 / 10(70 \%)$ & $1 / 10(10 \%)$ \\
Layer & $\mathrm{F}$ & $28 \mathrm{w}$ & $0 / 14$ & $3 / 14(21 \%)$ & $1 / 14(7 \%)$ \\
Layer & $\mathrm{G}$ & $28 \mathrm{w}$ & $1 / 14(7 \%)$ & $13 / 14(93 \%)$ & $3 / 14(21 \%)$ \\
Layer & $\mathrm{H}$ & $28 \mathrm{w}$ & $0 / 14$ & $10 / 14(71 \%)$ & $1 / 14(7 \%)$ \\
Layer & $\mathrm{I}$ & $28 \mathrm{w}$ & $0 / 11$ & $3 / 11(18 \%)$ & $0 / 11$ \\
Layer & $\mathrm{J}$ & $28 \mathrm{w}$ & $0 / 15$ & $6 / 15(40 \%)$ & $0 / 15$ \\
Layer & $\mathrm{K}$ & $28 \mathrm{w}$ & $3 / 13(23 \%)$ & $9 / 13(69 \%)$ & $4 / 13(31 \%)$ \\
Layer & $\mathrm{L}$ & $28 \mathrm{w}$ & $2 / 15(13 \%)$ & $6 / 14(43 \%)$ & $1 / 15(7 \%)$ \\
\hline
\end{tabular}

${ }^{a}$ Number of positive sample/number of birds tested per flock (percent positive samples in flocks with positive sera) 


\section{Figure legends}

Figure 1. Schematic representation of the avian astrovirus genome and information on and location of the PCR primers used. (A) Schematic representation of the avian astrovirus genome. The four different primer sets and the primer used for reverse transcription (RT) are indicated by arrows and nucleotide numbers. The astrovirus genome consists of ORF-1a, ORF-1b and ORF-2. Nucleotide numbers in the boxes indicate the start and stop sites. (B) Primer names, genome location, sequence, specificity for the known avian astroviruses, product length (in base pairs: bp) and annealing temperature $\left({ }^{\circ} \mathrm{C}\right)$ used for the PCR reaction, and PCR results with isolate 19 are indicated.

Figure 2. Nucleotide sequence alignment of the ORF-1a gene (nucleotides 2207-2608,) sequences of the novel avian nephritis virus-3 isolates and the avian nephritis virus-1. The 12 nt insert specific for the new isolates is indicated in bold.

Figure 3. Amino acid sequence alignment of the deduced sequence of the ORF-1a gene of the novel avian nephritis virus-3 isolates and avian nephritis virus1. The 4 amino acid insert specific for the new isolates is indicated in bold.

Figure 4. Phylogenetic tree of the ORF1A sequence of the avian nephritis virus-3 isolates and reference sequences. Shown is a phylogenetic tree of the 407 nucleotide long sequences of the ORF-1 a of all 10 isolates of the newly isolated avian nephritis viruses, ANV-1 and turkey astrovirus-1 (TAstV1). All GenBank accession numbers are indicated in Table 1. The phylogenetic tree was constructed by the neighbour-joining method and bootstrapped a 1000 times using Bioedit and Mega4.1. Values are indicated in the tree. 
Figure 1

A

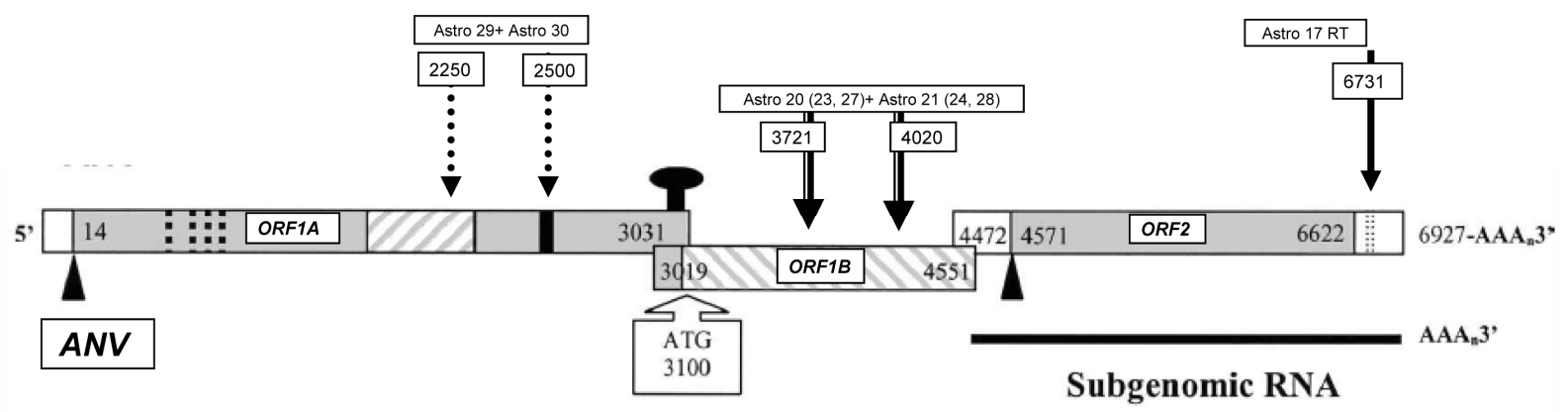

B

\begin{tabular}{|c|c|c|c|c|c|c|c|}
\hline Primer & & Location & Sequence 5'->3' & Specificity & $\begin{array}{l}\text { Length } \\
\text { product (bp) }\end{array}$ & $\begin{array}{l}\text { Annealing } \\
\text { temperature }\end{array}$ & $\begin{array}{l}\text { PCR product } \\
\text { isolate } 19\end{array}$ \\
\hline Astro 20 & $\mathrm{FW}^{\mathrm{a}}$ & ORF1b & TGG HCM CCY TTY TTY GGH G & \multirow{2}{*}{$\begin{array}{c}\text { ANV-1, CAstV and } \\
\text { ANV3 }\end{array}$} & \multirow{2}{*}{300} & \multirow{2}{*}{48} & \multirow{2}{*}{$+^{\mathrm{c}}$} \\
\hline Astro 21 & $\mathrm{REV}^{\mathrm{b}}$ & ORF1b & RTT RTC MAC DGT KGT DGA RWA YTG & & & & \\
\hline Astro 23 & FW & ORF1b & TGG ACC CCC TTC TTT GGT & \multirow[b]{2}{*}{ ANV-1 and ANV-3 } & \multirow[b]{2}{*}{300} & \multirow[b]{2}{*}{46} & \multirow[b]{2}{*}{+} \\
\hline Astro 24 & REV & ORF1b & ATT ATC AAC AGT TGT TGA AAA TTG & & & & \\
\hline Astro 27 & FW & ORFf1b & TGG ACC CCC TTC TTT GGT GG & \multirow{2}{*}{ CAstV } & \multirow{2}{*}{359} & \multirow{2}{*}{55} & \multirow{2}{*}{$-{ }^{d}$} \\
\hline Astro 28 & REV & ORF1b & TGY TTR CGG TGG AGC CAT GC & & & & \\
\hline Astro 29 & FW & ORF1a & GTY CTY ACC GAR GAR GAR TAY C & \multirow{2}{*}{ ANV-3 } & \multirow{2}{*}{260} & \multirow{2}{*}{51} & \multirow{2}{*}{+} \\
\hline Astro 30 & REV & ORFf1a & AAD GTT ATY CTC CTA RGB TKH C & & & & \\
\hline Astro 17 & $\mathrm{RT}^{\mathrm{e}}$ & $3^{\prime}-\mathrm{UTR}^{\mathrm{f}}$ & TCG WTS CTA CYC & astrovirus & NA & & \\
\hline
\end{tabular}

Figure 1. Schematic representation of the avian astrovirus genome and information on and location of the PCR primers used. (A) Schematic representation of the avian astrovirus genome. The four different primer sets and the primer used for reverse transcription $(R T)$ are indicated by arrows and nucleotide numbers. The astrovirus genome consists of ORF-1a, ORF-1b and ORF-2. Nucleotide numbers in the boxes indicate the start and stop sites. (B) Primer names, genome location, sequence, specificity for the known avian astroviruses, product length (in base pairs: bp) and annealing temperature $\left({ }^{\circ} \mathrm{C}\right)$ used for the PCR reaction, and PCR results with isolate 19 are indicated.

${ }^{\text {a }} \mathrm{FW}$ : forward.

${ }^{\mathrm{b}} \mathrm{REV}$ : reverse.

${ }^{\mathrm{c}}+$ PCR product present.

d - No PCR product detected.

${ }^{\mathrm{e}} \mathrm{RT}$ : reverse transcription.

f 3'-UTR: 3'-untranslated region. 
Isolate19 1 CACAAAACTAAATCGATCTCAAAAGCTGCCTTCATGAAAACAAAAGTCCTTACCGAAGAAGAATACCGTC

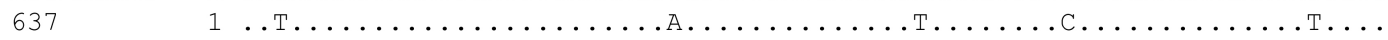

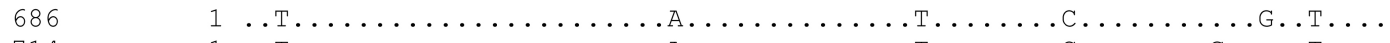

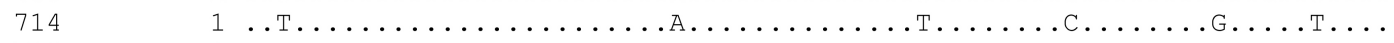

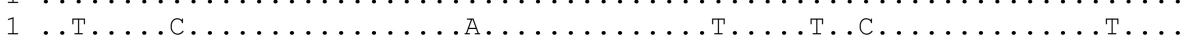

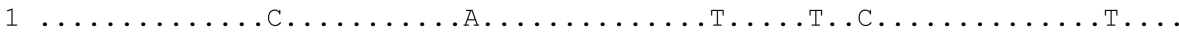

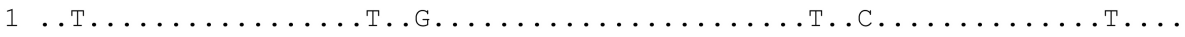

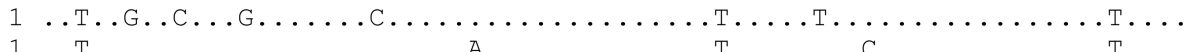
2207 ....G.C..G....T.G.............................

Isolate19 71 GGTTAGAGGAAGAAGGCTTCTCAAAAGATGAGATTAAAGAGATCGTGGACAATCTGCGAGAACAAGCCTG

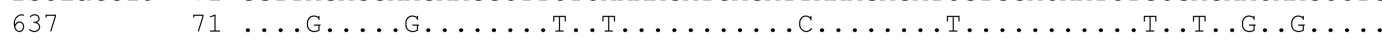
$686 \quad 71 \ldots$.

Isolate19 141 GATCGATTACCAGAATCAGCTAGATGAAGAAGGTGATGATGACTGGTATGAGCAAATGACTGAAGATCAA

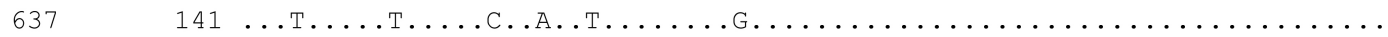

$686 \quad 141 \ldots$ т. с. . . . .А.

$714 \quad 141 \ldots$ T...

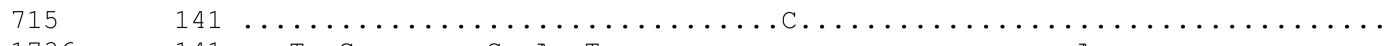

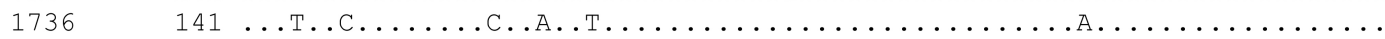

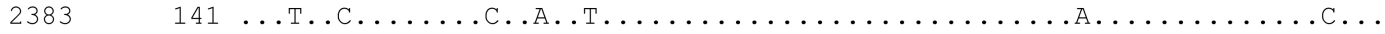

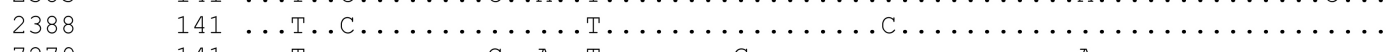

Isolate19 211 AGAATTAATGATGAGATTGATAAGCAAATTGAGCAAGACCTTGAAGATCGAGGAGAATGGTATGGCCAGT

$637 \quad 211 \ldots$. . C . . . . . . . . . . . . . . . . . . . . . . . . . . . . .

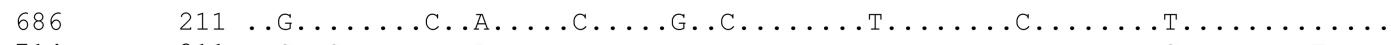

$714 \quad 211 \ldots$ G. . . . . . . . .

715

1736

2383

2388

7279

161317

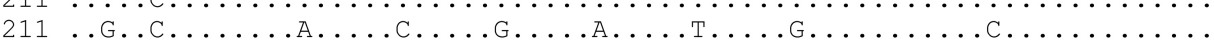
211 ............................................. $211 \ldots$......................GAGT.................... $211 \ldots$. . . . . . . . .А. . . . . . ANV1

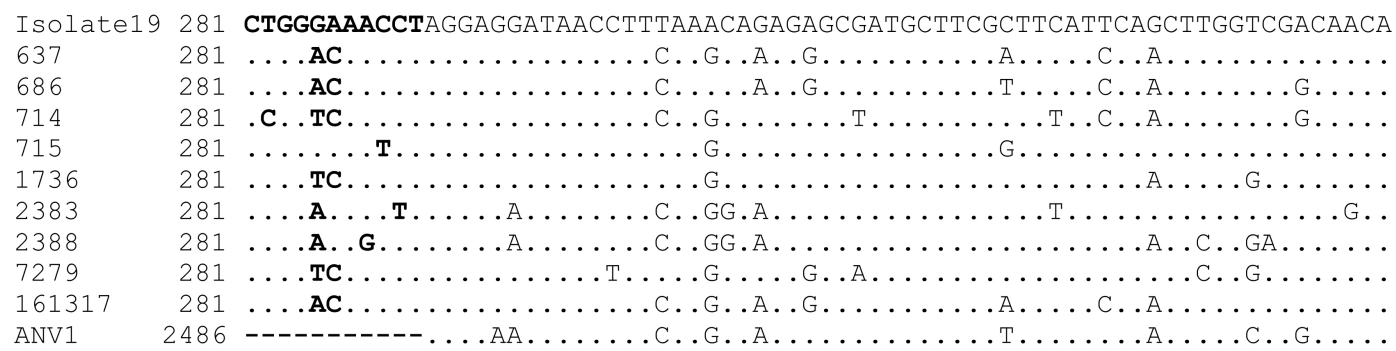

\begin{tabular}{|c|c|c|}
\hline Isolate19 & 351 & ACAAATAGCCACAATTTCATTTCCTGATGGCTATGAAGATAGAGCTGAAGAACTCTATAATAA \\
\hline 637 & 351 & \\
\hline 686 & 351 & . \\
\hline 714 & 351 & ..... . . \\
\hline 715 & 351 & \\
\hline 1736 & 351 & 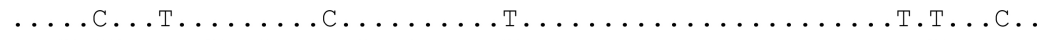 \\
\hline 2383 & 351 & ..T.CG...T..C...С..А. \\
\hline 238 & 35 & $\ldots \ldots \ldots \ldots \ldots \ldots \ldots$ C..... \\
\hline 7279 & 351 & $\ldots \ldots$ СG. \\
\hline 161317 & 351 & ....... \\
\hline & & T \\
\hline
\end{tabular}

Figure 2. Nucleotide sequence alignment of the ORF-1 a gene (nucleotides 2207-2608,) sequences of the novel avian nephritis virus-3 isolates and the avian nephritis virus-1. The 12 nt insert specific for the new isolates is indicated in bold. 


Isolate19
637
686
714
715
1736
2383
2388
7279
161317
ANV1-orfla

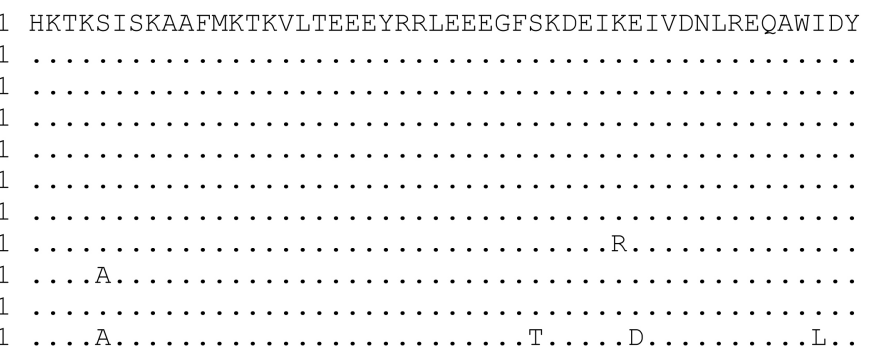

Isolate19

51 QNQLDEEGDDDWYEQMTEDQRINDEIDKQIEQDLEDRGEWYGQSGKPRRI

637

686

714

715

1736

2383

2388

7279

161317

ANV1-orf1a

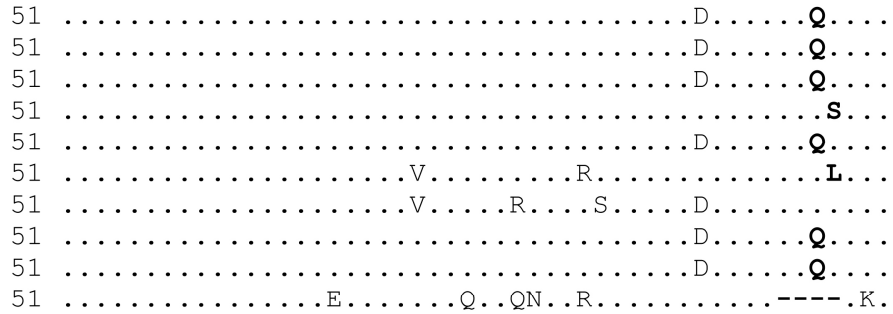

Isolate19
637
686
714
715
1736
2383
2388
7279
161317
ANV1-orf1a

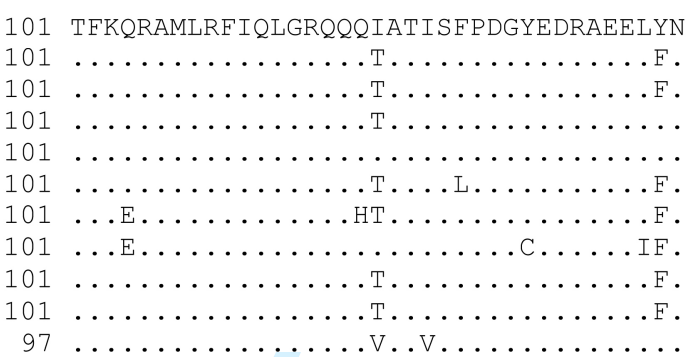

Figure 3. Amino acid sequence alignment of the deduced sequence of the ORF-1a gene of the novel avian nephritis virus-3 isolates and avian nephritis virus-1. The 4 amino acid insert specific for the new isolates is indicated in bold. 


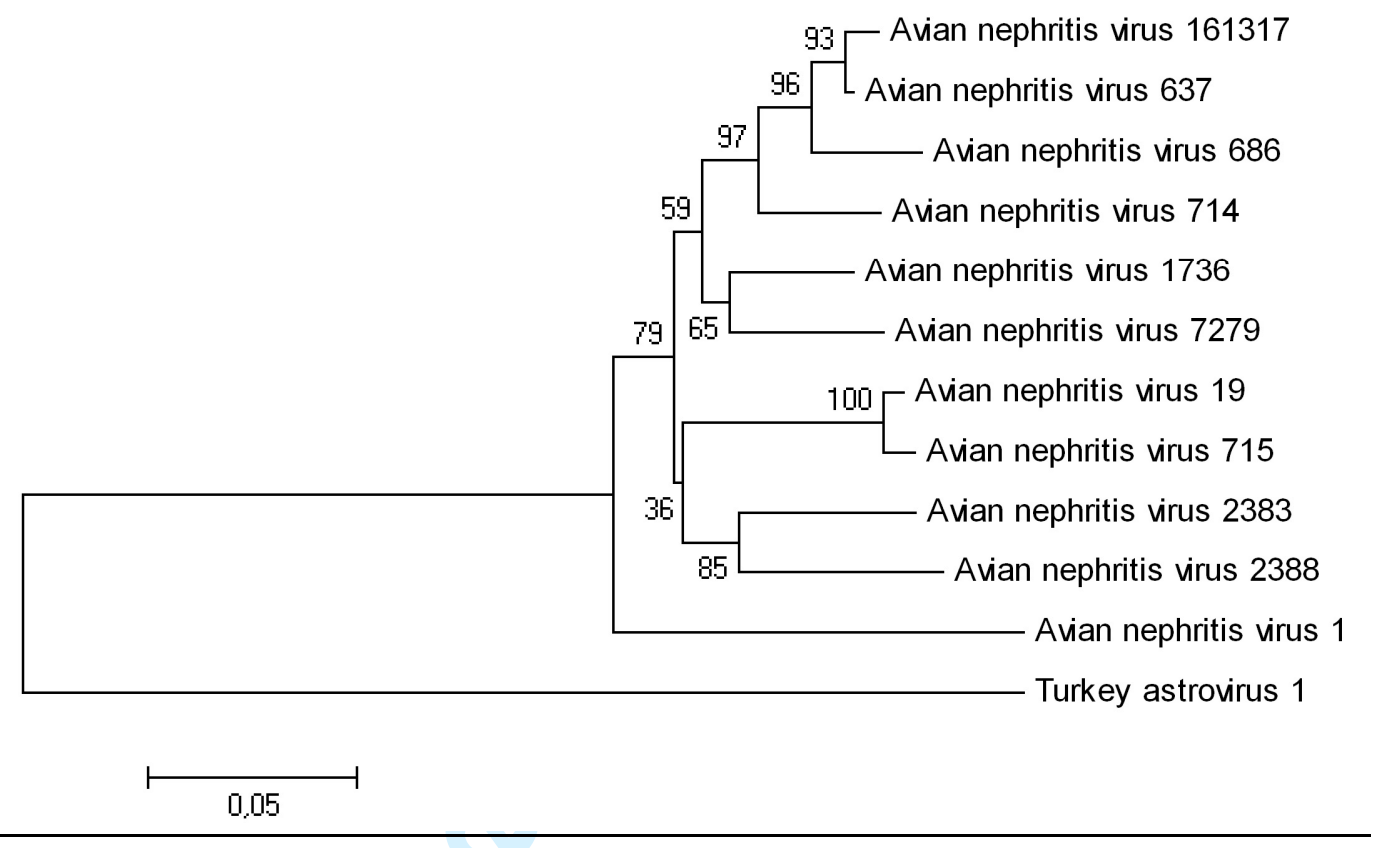

Figure 4. Phylogenetic tree of the ORF1A sequence of the avian nephritis virus-3 isolates and reference sequences. Shown is a phylogenetic tree of the 407 nucleotide long sequences of the ORFla of all 10 isolates of the newly isolated avian nephritis viruses, ANV-1 and turkey astrovirus-1 (TAstV1). All GenBank accession numbers are indicated in Table 1. The phylogenetic tree was constructed by the neighbour-joining method and bootstrapped a 1000 times using Bioedit and Mega4.1. Values are indicated in the tree. 